\title{
Delayed presentation and increased prevalence of proliferative vitreoretinopathy for primary rhegmatogenous retinal detachments presenting during the COVID-19 pandemic lockdown
}

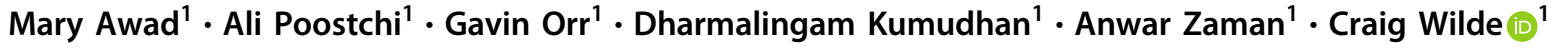

Received: 26 May 2020 / Accepted: 18 June 2020 / Published online: 29 June 2020

(c) The Royal College of Ophthalmologists 2020

We read with interest your recent article 'The impact of COVID policies on acute ophthalmology servicesexperiences from Moorfields Eye Hospital NHS Foundation Trust' [1]. Interestingly you report reduced attendance with new retinal detachments (RD) compared with the same period last year. As you state, the reasons for such a decrease could be multiple. It would be interesting to know how much of the reduction, if any, represents declining out of area referrals in the face of social distancing and perceived travel restrictions. Your findings may reflect a sudden cessation in referrals from regions further afield. In Nottingham, we have undertaken a detailed analysis of our rhegmatogenous $\mathrm{RD}$ cases and would like to share our rational for undertaking such an analysis. Our findings are reported below.

The spread of coronavirus disease 2019 (COVID-19) has caused disruption to many aspects of life. Social distancing became the primary response to reduce infection. The slogan 'stay home, protect the NHS, save lives' became well recognised. The idea was simple: slow transmission to protect hospital capacity, preventing resource overload, maintaining effective healthcare systems with ventilator/ staff availability. Routine surgery/outpatients were cancelled. Some ophthalmologists were redeployed. The direct impact of COVID-19 is clear, measured in terms of thousands of tragic deaths. Indirect consequences on public health are covert and yet to manifest.

There are concerns regarding potential decreased cancer survival in the near future. Screening/diagnostic procedures are delayed with rapid access oncology clinic referrals decreasing [2, 3]. Apprehension cancers will become

Craig Wilde

craig_wilde@hotmail.com

1 Ophthalmology Department, Queen's Medical Centre, Nottingham, UK inoperable exists. Similarly, outcomes for RD are influenced by early presentation [4]. With this in mind, we explored whether lockdown delayed presentations for RD, increasing rate of proliferative vitreoretinopathy (PVR).

Retrospective case note review was conducted for all consecutive patients undergoing primary RD surgery at our tertiary vitreoretinal centre between 23/10/2019 and 19/05/ 2020. Patients were divided into two groups: 5-months prior to and 2-months following lockdown (23/03/2020) and compared for differences in presenting features. Statistical analysis was performed using SPSS (Mac) v24 (SPSS, inc, Chicago, Illinois, USA).

Pre-lockdown 92 RD presented, while post-lockdown there were 37. Mean ages were similar [59.5 (17-89) vs 58.8 (28-89) years respectively]. Following lockdown mean symptom duration increased [14.8 days (SD 25.6) vs 22.5 days (SD 42.0); $p=0.2297$ ] and proportion of patients with macula-on RD decreased (47.8 vs $32.4 \%, p=0.1210$ ). There was a statistically significant increase in cases presenting with PVR (9.8 vs $24.3 \%, p=0.0471)$. Cases requiring silicone oil endotamponade or encirclement or both increased (35.3 vs $40.5 \%, p=0.1378$ ) (Fig. 1).

Primary surgery for RD has reached $90 \%$ success rate. The most important factor determining visual prognosis is macula detachment. The leading cause of failure is PVR [5]. We suggest the lockdown may have delayed presentations to hospital for RD. COVID-19 presents barriers to accessing healthcare. Optometrists and GP's (the first port of call) are less accessible. Patients may seek help later through concern of contracting the virus. This hypothesis is supported by the trend for a longer duration of symptoms prior to presentation and increased proportion of macula-off $\mathrm{RD}$, although the differences did not reach statistical significance. This is important as the duration of RD is a risk factor for PVR.

Prevalence of PVR at presentation varies (3.9-13.7\% in developed countries) [5]. Rates appear higher in developing countries where suboptimal access to healthcare is reported, 


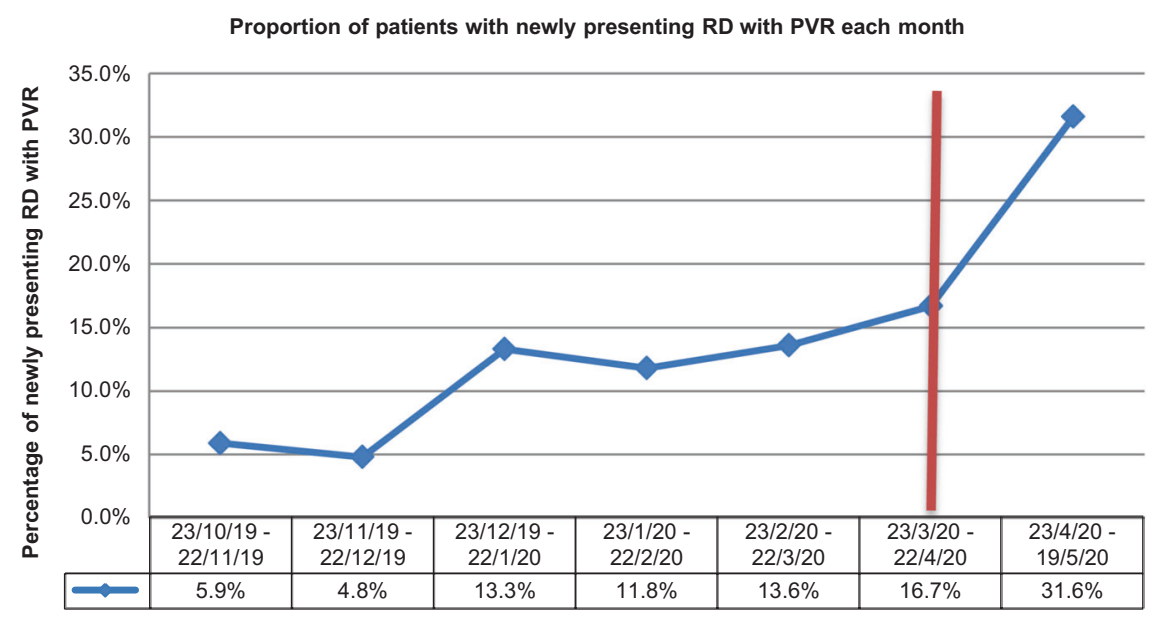

Fig. 1 Line chart showing the percentage of newly presenting rhegmatogenous retinal detachments that have proliferative vitreoretinopathy (blue line). Data are plotted for each month, covering the period of study between 23/10/19 until 19/5/20. The vertical red line indicates the time point at which the United Kingdom lockdown was officially announced (23/3/20). Following this date, a spike in the prevalence of proliferative vitreoretinopathy is clearly seen among newly presenting retinal detachments, $p=0.0471$. including Venezuela, where 26.9\% have grade C PVR [5]. Our study suggests COVID-19 may have caused, through delayed presentation, increased prevalence of PVR, that appears comparable with less developed healthcare systems. These patients will likely require multiple, complex surgeries with higher chance of failure and worse visual outcomes.

It is essential patients seek treatment promptly for nonCOVID-19 illnesses. Prioritising COVID-19 and the associated message to stay at home can introduce other morbidities that can themselves cause harm.

\section{Compliance with ethical standards}

Conflict of interest The authors declare that they have no conflict of interest.

Publisher's note Springer Nature remains neutral with regard to jurisdictional claims in published maps and institutional affiliations.

\section{References}

1. Wickham L, Hay G, Hamilton R et al. The impact of COVID policies on acute ophthalmology services-experiences from Moorfields Eye Hospital NHS Foundation Trust. Eye 2020. https:// doi.org/10.1038/s41433-020-0957-2.

2. Dinmohamed AG, Visser O, Verhoeven RHA, Louwman MWJ, van Nederveen FH, Willems SM, et al. Fewer cancer diagnoses during the COVID-19 epidemic in the Netherlands. Lancet Oncol. 2020;S14702045:30265-5. https://doi.org/10.1016/S1470-2045(20)30265-5.

3. Jones D, Neal RD, Duffy SRG, Scott SE, Whitaker KL, Brain K. Impact of the COVID-19 pandemic on the symptomatic diagnosis of cancer: the view from primary care. Lancet Oncol. 2020. https:// doi.org/10.1016/S1470-2045(20)30242-4

4. Park DH, Choi KS, Sun HJ, Lee SJ. Factors associated with visual outcome after macula-off rhegmatogenous retinal detachment surgery. Retina 2018;38:137-47.

5. Tseng W, Cortez RT, Ramirez G, Stinnett S, Jaffe GJ. Prevalence and risk factors for proliferative vitreoretinopathy in eyes with rhegmatogenous retinal detachment but no previous vitreoretinal surgery. Am J Ophthalmol. 2004;137:1105-15. 